\title{
D e l'utilité de la pédagogie pour la formation continue des médecins
}

Le travail des enseignants est toujours un travail difficile. Ceux qui prennent en charge la formation continue des médecins cumulent les difficultés:

- Formation d'adultes pour laquelle on ne peut plus fair re l'impasse sur les croyances et les connaissances antérieures qui, vraies ou fausses, sont fortement ancrées par des années d'une « expérience professionnelle » accumulé au hasard des innombrables relations duelles médecin/malade dont aucune n'est semblable à l'autre.

- H étérogénéité de groupes formés au sein d'une profession qui est structurellement profondément individualiste.

- Absence de programmes imposés par une structure administrative et derrière lesquels on peut se réfugier en cas de désaccord des participants avec les objectifs proposés.

- O rganisation des ensei gnements dans des condi tions parfois contraignantes, le soir ou hors des heures «normales", après une semaine de travail harassante.

- Exigence des participants de recevoir le maximum de connaissances « utiles» dans le minimum de temps.

- Formation à des tâches professionnelles complexes nécessitant un enseignement personnalisé $\mathrm{N}$ os systèmes de soins sont divers de part et d'autre del'Atlantique ou au sein de notre vieille Europe. De la même façon, les expériences de formation médicales continues sont, elles aussi, variées, marquées par la diversité des organisateurs, des financeurs, des modes de fonctionnement professionnels des médecins, de la culture et de l'histoi re propres de chaque pays. Les expériences accumulées, les échecs et les réussites des uns et des autres constituent une richesse importante même si les divers tés de contextes les rendent souvent impossibles à transposer telles quelles et même difficilement transposables.

La formation continue des médecins est plus que toute autre susceptible de bénéficier de la réflexion et des outils pédagogiques modernes. La Revue qui naît aujourd'hui, PÉDAGOGIE MÉDICALE, doit pouvoir devenir pour tous les francophones, tout à la fois le lieu d'échange d'expériences, l'outil de vulgarisation pédagogique, l'instrument transdisciplinaire de réflexion prospective dont la formation continue médicale a besoin.

1. Un lieu d'échanges d'expérience.

Réaliser un prototype d'action pédagogique est bien. L'écrire permet de fixer l'expérience acquise. Le publier est une occasion de le soumettre à la réflexion critique des autres acteurs de terrain. Enregistrer les réactions de ceux qui se sont confrontés dans des circonstances différentes à des problèmes analogues ne peut que stimuler chez tous l'imagination, la réflexion et l'élaboration de nouveaux prototypes. II est intéressant de comprendre pourquoi telle expérience qui est possible 
ici se heurte ailleurs à des difficultés. Cela permet de résoudre celles-ci et d'analyser les limites de ce qui est ainsi proposé La reconnaissance d'une réalisation de qualité est peut-être plus importante quand elle vient del'étranger : "Nul n'est prophète en son pays... »

2. Un outil de vulgarisation pédagogique. "Qui peut faire, fait. Qui ne sait pas faire enseigne. Q ui ne sait pas enseigner fait de la pédagogie ». Voilà comment aujourd'hui encore, certains aiment à brocarder les chercheurs en pédagogie, les suspectant de se complaire dans des joutes oratoires déconnectées de tout pragmatisme. Les responsables de la formation continue sont pour la plupart des médecins en exercice. Ce sont également des enseignants. Pour l'extrême majorité d'entre eux, ils manquent de la disponibilité nécessaire pour suivre les évol utions de cette science de l'éducation pourtant si dynamique et, parfoissi éclairante des difficultés auxquelles nous avons à faire face. II est de la responsabilité des dirigeants de FM C d'interpeller les pédagogues sur les problèmes qu'ils rencontrent. II est de la responsabilité des pédagogues de signaler aux dirigeant deFM C les nouveaux outils, les nouvelles approches dont ils pourraient tirer profit. Encore faut-il qu'il y ait un lieu pour que cette rencontre et ce dialogue soit organisés. PÉDAGOGIE MÉDICALE devra jouer ce rôle.

3. Un instrument transdisciplinaire de réflexion pros pective.

La réflexion pédagogique médicale évolue sans cesse et de nouveaux outils sont régulièrement proposés. Elle a déjà amplement bénéficié dela rencontre des médecins et des professeurs de médecine avec des spécialistes de sciences humaines. N ous avons ainsi tiré profit des apports successifs de la pédagogie des adultes, de la pédagogie par objectifs, de l'émergence de la dynamique de groupe, des avancées dans les techniques de communication de la psychologie classique, de la psychologie expérimentale, plus récemment de la psychologie cognitive. Toutes ces approches successives, convergentes, différentes ont enrichi notre réflexion pédagogique. Elles nous ont permis de mieux répondre à la question de savoir comment mieux enseigner : Q uelles connai ssances déclaratives, gestuelles, relationnelles faut-il transmettre aux futurs médecins ou aux médecins installés ? Comment les transmettre? $\mathrm{H}$ ier, ceux qui développaient la psychologie cognitive nous ont permis de mieux comprendre comment est appris ce qui est enseigné et nous ont ouvert d'autres perspectives, nous permettant de mieux aider à apprendre : Comment favoriser les apprentissages? Comment sassurer qu'ils seront effectivement utilisés, introduits dans la pratique médicale?

Demain, c'est un nouveau changement complet de paradigme que nous aurons à affronter. Les connais sances médicales deviennent si nombreuses et évoluent si rapidement qu'il est illusoire de vouloir les engranger, les mémoriser et les utiliser toutes. Parallèlement, les progrès fulgurants de l'informatique et de la télé matique sont tels qu'ils sont susceptibles de mettre à disposition de chaque médecin, en temps réel, toute l'information actualisée dont il a besoin pour prendre une décision face à un de ces patients. La question n'est donc plus de savoir quelles informations dispenser mais quels processus de recherche d'informations, quelles capacités d'utilisation de ces informations il convient de développer chez les médecins. Pour ces nouveaux challenges, c'est, avec les spécialistes des sciences humaines, de la collaboration des tous les métiers de l'informati que dont nous aurons besoin. Les M édecins exi gent de leurs responsables une formation continue performante, ludique et efficiente. Pour la leur offrir, nous devons posséder un outil de rencontres, d'échanges et de prospectives. PÉDAGOGIE MÉDICALE constitue une opportunité exceptionnelle pour le créer. Elle le sera si nous le voulons et si nous y travaillons. Tous.

Charles H ON N ORAT, Rennes, France 